\title{
REVIEWS OF BOOKS
}

Chemotherapy of Gonococcic Infections. Russell D. Herrold, B.S., M.D., Associate Professor of Surgery (Urology), College of Medicine, University of Illinois. $137 \mathrm{pp}$. Henry Kimpton. London, 1943. Price 15s.

Dr. Herrold tells us that there are well over a million new infections of gonorrhoea each year. He therefore concludes that there is room for a monograph that deals with 'the management of these infections through the avenue of chemotherapy'. There are few venereologists who will not agree with this statement, as there is certainly today no book in the English language dealing effectively with the modern treatment of this disease.

Dr. Herrold lays stress on the importance of all doctors being acquainted with epidemiological measures in combating venereal diseases and in making prophylactic centres available for the civil population. The early history in the use of the original prontosil and of sulphanilamide in the treatment of gonorrhoea is given in detail, the author having been one of the first to use the latter. He dismisses urethroscopy as rarely necessary, but there must be many workers who will disagree with him in this and maintain, in my opinion rightly, that anterior urethroscopy should be carried out as a test for cure in all male case, in order to eliminate the possibility of stricture developing in later life. It is pleasing to note that he does not even mention the complement fixation test for gonorrhoea, but I consider that he gives too much importance to microscopic and cultural investigations of the centrifuged deposit of the urine, and that gonococci are more likely to be detected in secretions obtained before the first morning micturition. He deals with cultural examinations in detail and emphasizes the dangers of false positive oxidase reactions, especially in the cases when it is impossible to obtain pure cultures. There is an excellent chapter on toxic reactions to sulphathiazole, but the author omits the fact that a further course with a different drug (for instance, sulphadiazine) does not often produce toxic symptoms in patients who have developed an allergic sensitization to sulphathiazole. The description of the carrier state and of the management of sulphonamide failures contains some valuable information, but he lays too much stress on the local treatment of the various complications which, in my experience, are invariably a consequence of drug resistance and should be treated by raising the patient's resistance with protein shock, mild or severe, followed, if necessary, by a further course of sulphonamide therapy.

This book can be warmly recommended, but nevertheless a classic on chemotherapy in the treatment of gonococcal infections has yet to be written.

Social Service in the Clinics for Venereal Diseases. 2nd Edition. By Dorothy Manchée. 119 pp. Baillière, Tindall \& Cox. London, 1943. Price 5s.

The second edition of Miss Manché's book is opportune at the present time when there is a marked increase of infections and also an expansion of treatment facilities. This book can be unreservedly commended to the attention of directors of clinics, medical officers of health and all those interested in the subject of venereal disease. The value of social services as an auxiliary to medical treatment has long been emphasized : a patient is primarily a human being whose disease must be considered in conjunction with his personal life, his surroundings and his problems. Failure to consider the social factors may lead to failure to achieve the expected benefit from purely medical treatment, to undue protraction of the ailment, or in the case of the venereal disease patient to premature discontinuance of therapy and spread of infection.

In no department of Medicine can a trained social worker be of greater value than in the venereal diseases clinic. The necessity of such appointments is unfortunately not universally recognized, partly because of the stress rightly laid on confidential treatment. Experience has amply shown, however, that the confidential aspects of medical treatment are not only not violated but are actually enhanced by the addition of a social worker to the clinic staff. Miss Manchée gives a simple straightforward account of the manifold duties of the venereal diseases almoner, the individual problems met with and the possible solutions. The aims are to smooth out difficulties preventing regularity of attendance or predisposing to default before completion of treatment, and to awaken in the individual the sense of personal and corporate social responsibility which lessens the risk of future infection. The alleviation of personal problems makes the task of medical treatment easier and more certain ; the rehabilitation of the individual is gained by sympathetic understanding of the underlying problems and by assistance in securing suitable employment. In the past rehabilitation has been considered almost exclusively in relation to prostitution, but it is now recognized that the provision of unobtrusive social assistance for the casually promiscuous is not of any less importance. It is a matter for regret that up-to-date social service in clinics has been confined chiefly to female patients and that provision has not been made for dealing with the equally urgent problems of the male patient.

The war-time disorientation of family life, the relaxation of parental responsibility, the direction of female labour into industry, the hours of work and the constant shifting of large numbers of the population have not only accentuated the existing difficulties but have created new problems in venereal diseases control. Although from the purely medical aspect the infectivity of syphilis or gonorrhoea can now be controlled rapidly by short-term intensive treatment this does not solve the difficulties of medical observation afterwards, of influencing the attendance for investigation and treatment of alleged sources of infection and subsequent contacts or of default on transfer from one centre to another. The shorter period of time during which the patient comes 